CONGENITAL HEART DISEASE

\title{
Changing practice of cardiac surgery in adult patients with congenital heart disease
}

\author{
S K Srinathan, R S Bonser, B Sethia, S A Thorne, W J Brawn, D J Barron
}

Heart 2004;91:207-212. doi: 10.1136/hrt.2003.032011

See end of article for authors' affiliations

Correspondence to: Dr Sadeesh K Srinathan, Birmingham Children's Hospital, Steelhouse Lane Birmingham B4 6NH, UK; sadeesh@macunlimited.net

Accepted 27 April 2004
Objectives: To review 13 years' data from a unit for grown ups with congenital heart disease (GUCH) to understand the change in surgical practice.

Methods: Records were reviewed of patients over 16 years of age undergoing surgery between 1 January 1990 and 31 December 2002 in a dedicated GUCH unit. Patients with atrial septal defects were included but not those with Marfan's syndrome or undergoing a first procedure for bicuspid aortic valves. Three equal time periods of 52 months were analysed.

Results: Of 474 operations performed, 162 (34.2\%) were repeat operations. The percentage of repeat operations increased from 24.8\% (41 of 165) in January 1990-April 1994 to $49.7 \%$ (74 of 149) in September 1998-December 2002. Mortality was 6.3\% ( $\mathrm{n}=30$ ). The median age decreased from 25.4 years (interquartile range 18.7) in January 1990-April 1994 to 23.9 (interquartile range 17.3) in September 1998-December 2002 ( $p=0.04$ ). The proportion of patients with a "simple" diagnosis decreased from 45.4\% (74 or 165) in January 1990-April 1994 to $27.5 \%$ (41 of 149) in September 1998-December 2002 ( $p=0.013$ ). Pulmonary valve replacements in operated tetralogy of Fallot increased from one case in January 1990-April 1994 to 23 cases in September 1998-December 2002 and conduit replacement increased from five cases to 17. However, secundum atrial septal defect closures decreased from 35 cases to 14 ( $p<0.0001$ ). The estimated cost (not including salaries and prosthetics) incurred by an adult patient with congenital heart disease was $£ 2290$ compared with $£ 2641$ for a patient undergoing coronary artery bypass grafting.

Conclusion: Despite the impact of interventional cardiology, the total number of surgical procedures remained unchanged. The complexity of the cases increased particularly with repeat surgery. Nevertheless, the patients do well with low mortality and the inpatient costs remain comparable with costs of surgery for acquired disease.
A dvances in paediatric heart surgery over the past few decades have led to increasing numbers of long term survivors from both palliative and corrective surgery. Currently $80-85 \%$ of patients born with congenital heart disease can expect to reach adulthood. ${ }^{1}$

This has led to an increasing population of adults who have had surgery as children in addition to patients who present as adults with congenital heart disease. There are now as many adults with congenital heart disease as children. ${ }^{2}$ This can be expected to continue with an estimated 167805 adults with congenital heart disease in the UK by $2010 .^{1}$

The changing nature of this population is of growing importance, especially with regard to provision of adequate services for these patients. Grown ups with congenital heart disease (GUCH) have complex needs and the services they require include cardiology, cardiac surgery, and a host of other related services ranging from specialised obstetric services to access to transplantation. ${ }^{2}$

To assess future needs, this study has been performed to review the changes in the GUCH population undergoing surgery in our unit since its inception in 1989.

We were interested in the following questions:

- Has the nature of patients undergoing surgery for congenital heart disease changed over the past 13 years in? Are there changes in their age or premorbid states?

- Has the type of conditions for which these patients undergo surgery changed?

- What are the changes in the procedures being performed during this time period?
- What are the outcomes in these patients and have these outcomes changed over this time period?

- What are the possible implications for the future provision of services?

\section{METHODS \\ Patients}

A database and charts of all patients over 16 years of age who underwent cardiac surgery from 1 January 1990 to 31 December 2002 were reviewed. This time period begins at the appointment of two dedicated adult congenital cardiac surgeons to the unit. The majority of operations were performed in an adult cardiac surgical unit by congenital heart surgeons, although some 16-18 year old patients were operated on at the Birmingham Children's Hospital, where the children's hospital environment had been felt to be more appropriate to the patients' requirements.

All patients in this study underwent operations for cardiac malformations diagnosed when they were children or adults. We included patients undergoing closure of secundum atrial septal defects in this study. However, patients undergoing an isolated first time aortic valve replacement with bicuspid valves were excluded as were patients with Marfan's syndrome undergoing a first procedure as an adult.

Abbreviations: $\mathrm{GUCH}$, grown up congenital heart disease; ITU, intensive treatment unit; NYHA, New York Heart Association; SASD secundum atrial septal defect 
In keeping with other studies bicuspid aortic valve disease and Marfan's syndrome fall within the remit of acquired cardiac surgery and are operated on routinely by adult cardiac surgeons specialising in these fields. These patients therefore do not enter the system through a GUCH programme, nor do they affect it.

The anatomical diagnosis of the congenital heart defects were classified into simple, moderate, and complex categories according to the modified Canadian consensus conference criteria as used by the Bethesda conference report ${ }^{3}$ (appendix 1). This reflects the complexity of the underlying diagnosis and not necessarily the complexity of the surgical procedures carried out to treat the condition.

To analyse the complexity of the operations themselves, the type of procedures and the cardiopulmonary bypass data were examined. Cardiopulmonary bypass data act as a marker of the technical demands of repeat procedures and complex reconstructions.

Detailed analysis of preoperative New York Heart Association (NYHA) class, complications (any event that probably extended hospital stay), intensive treatment unit (ITU) stay (nights spent), and blood use (units of packed cells) was based only on data from patients operated on at the Queen Elizabeth Hospital.

\section{Data analysis}

The data were analysed after dividing the group into three equal time periods (eras) of 52 months. Era 1 was from l January 1990 to 30 April 1994, era 2 was from 1 May 1994 to 31 August 1998, and era 3 was from 1 September 1998 to 31 December 2002.

Continuous variables were analysed by analysis of variance or by the Kruskal-Wallis rank tests depending on normality and equality of variance in the variables. Categorical variables were compared by contingency table analysis. The analysis was carried out with Statview version 4.01 (Abacus Concepts, Berkeley, California, USA) on an Apple Macintosh computer.

The reported $\mathrm{p}$ values are the overall $\mathrm{p}$ value across the three eras. Where this is significant $(p<0.05)$, the individual $\mathrm{p}$ values between eras is reported if applicable. In the case of contingency table analysis, appendix 2 gives the individual cell $\chi^{2}$ values for significant results.

\section{RESULTS}

Over the study period 474 procedures were performed on 438 patients (table 1). There were 30 deaths (surgical mortality $6.3 \%)$. There were 162 repeat procedures $(34.2 \%)$. The median age was 25.4 years (interquartile range 18.7 years). The proportion of male to female patients was $55.3 \%$ to
44.7\%. The median hospital stay was six days (interquartile range 3 days) and the median ITU stay was one night (interquartile range 0 nights). Throughout the study period, the number of cases remained constant. However, there was a steady increase in the percentage of repeat operations with half of the operations $(49.7 \%)$ in the third era being repeat procedures. Patients were more symptomatic with an increase in the proportion of patients in NYHA class III and IV in the second and third eras $(p=0.0456)$ (table 1$)$.

There is a suggestion of patients becoming younger with the median age decreasing by 1.5 years between era 1 and era 3. However, this is a small difference although significant ( $p=0.0437$, Kruskal-Wallis rank test $)$.

Patients undergoing operation presented with increasingly complex defects with the percentage of simple diagnosis decreasing from close to half the cases to less than a third between era 1 and era 3 (fig 1, table 1). In contrast, moderate and complex conditions increased from $26.4 \%$ and $28.2 \%$ to $40.3 \%$ and $32.2 \%$ of cases, respectively ( $p=0.0134$ ).

The operative procedures being performed also increased in complexity (fig 2). This is most apparent in the major rise in the proportion of conduit and pulmonary valve replacements. Concurrently there was a major decrease in the number of surgically closed secundum atrial septal defects.

In the first era, SASDs made up nearly $40 \%$ of the surgical workload whereas pulmonary valve replacements and conduit replacements combined totalled less than 10\%. In contrast, in the third era, SASD repairs made up only $15 \%$ of cases and pulmonary valves made up a quarter of the cases and conduit close to $20 \%$. Operations on the left ventricular outflow tract continued to make up about one third of the cases.

The increase in conduit replacement was accounted for mainly by patients who previously had corrected pulmonary atresia and truncus arteriosus (13 of 27; 48\%). Patients undergoing pulmonary valve replacements predominantly had previously repaired tetralogy of Fallot and pulmonary regurgitation ( 18 of $30 ; 60 \%$ ).

When the cardiopulmonary bypass time and aortic cross clamp were compared, we found that this had nearly doubled since the beginning of the study period $(\mathrm{p}<0.001)$ (table 2$)$.

The results have remained constant over time (table 2 ). Mortality, complication rate, and ITU and hospital stay have remained unchanged. There was no change in blood use despite the increase in repeat procedures over time.

On the basis of the hospital costing for theatre usage, bed occupancy, drugs, and ITU facilities, the cost for GUCH was compared with that for coronary artery surgery in the same unit. Coronary artery bypass grafting (CABG) costs about

\begin{tabular}{|c|c|c|c|c|c|}
\hline & All eras & $\begin{array}{l}\text { Era } 1(1 / 1 / 90- \\
30 / 4 / 94)\end{array}$ & $\begin{array}{l}\text { Era } 2(1 / 5 / 94- \\
31 / 8 / 98)\end{array}$ & $\begin{array}{l}\text { Era } 3(1 / 8 / 98- \\
31 / 12 / 02)\end{array}$ & p Value* \\
\hline Number of procedures & 474 & 165 & 159 & 149 & \\
\hline Number of patients & 438 & 151 & 148 & 139 & \\
\hline Women & $212(44.7 \%)$ & $84(50.9 \%)$ & $60(37.7 \%)$ & $68(45.6 \%)$ & $0.0567 \dagger$ \\
\hline Men & $262(55.3 \%)$ & $81(49.1 \%)$ & $99(62.3 \%)$ & $81(54.4 \%)$ & \\
\hline Age (years) & $25.4(18.7)$ & $25.4(20.7)$ & $27.0(18.7)$ & $23.9(17.3)$ & $0.0437 \ddagger$ \\
\hline $\begin{array}{l}\text { Repeat procedures } \\
\text { NYHA class }\end{array}$ & $162(34.2 \%)$ & $41(24.8 \%)$ & $47(29.6 \%)$ & $74(49.7 \%)$ & $<0.0001 \dagger \S$ \\
\hline 1 & $2(0.7 \%)$ & $2(2.6 \%)$ & $0(0.0 \%)$ & $0(0.0 \%)$ & $0.0456+$ \\
\hline ॥ & $92(33.3 \%)$ & $35(44.9 \%)$ & $31(31.6 \%)$ & $26(26.3 \%)$ & \\
\hline III & 124 (44.9\%) & 28 (35.9\%) & $46(46.9 \%)$ & $50(50.5 \%)$ & \\
\hline IV & $58(21.0 \%)$ & $13(16.7 \%)$ & $21(21.4 \%)$ & $23(23.2 \%)$ & \\
\hline
\end{tabular}

Data are median (interquartile range) or number (\%).

*For differences across the three time eras; $\dagger \chi^{2}$ test; $\ddagger$ Kruskal-Wallis rank test; §era $3 v$ era 1 and era $3 v$ era 1 , $\mathrm{p}<0.001$; 9 more patients were in New York Heart Association (NYHA) class I and II and fewer in NYHA class III and IV in era 1 than in eras 2 and 3 (appendix 2). 

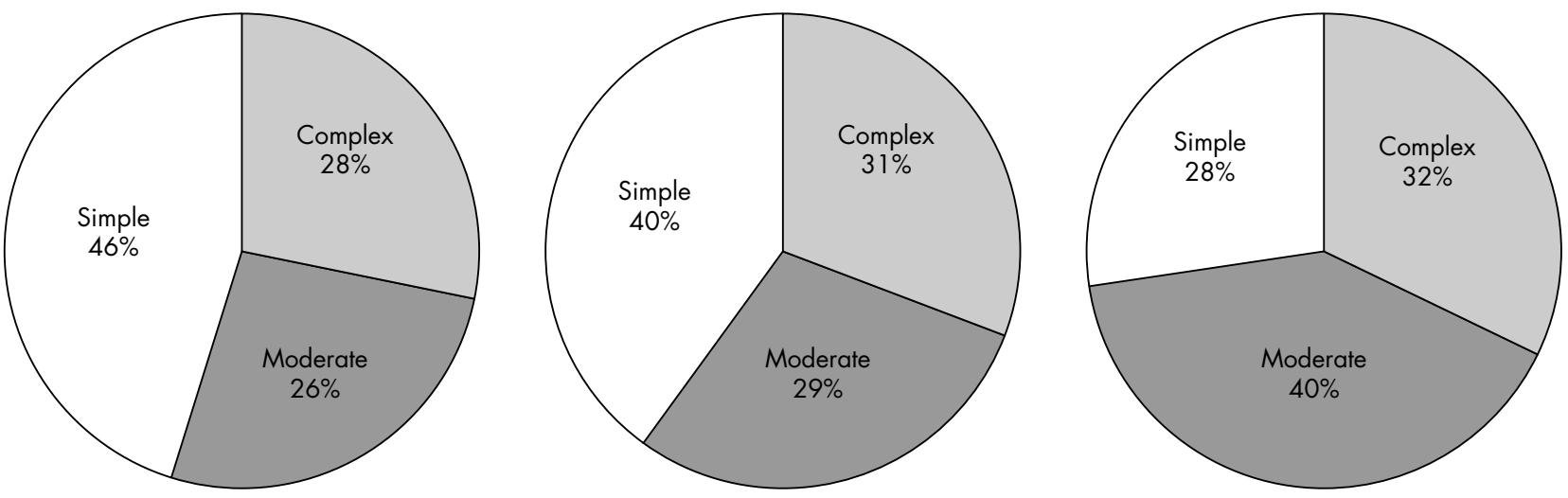

Figure 1 Distribution of diagnostic classes across the three eras. Class is defined according to the modified Canadian consensus conference criteria as used by the Bethesda conference report ${ }^{3}$ (appendix 1). The proportion of patients undergoing surgery who had a condition of moderate complexity (for example, tetralogy of Fallot) increased, whereas simple conditions (for example, secundum atrial septal defects) decreased and complex conditions (for example, transpositions of great vessels) remained unchanged. The difference in distribution of these cases across the three eras is significant $(p=0.0134)$, with the differences accounted for by the decrease in simple cases and increase in moderate cases between era 2 and era 3 .

$£ 2650$ excluding salary costs. A GUCH procedure costs $£ 2300$ excluding salary costs and valves.

\section{DISCUSSION}

The success of surgery for congenital heart disease has led to the rapid expansion in the numbers of patients with adult congenital heart disease. ${ }^{1}$ Many of these patients need lifelong follow up and may need repeat operations and reinterventions that have created challenges of their own and led to the development of a new subspecialty of adult congenital heart disease surgery. These adult patients, many of whom have undergone some type of corrective or palliative procedure, are now facing the long term effect of both the residua of the congenital defect and the sequelae of the treatment. ${ }^{4}$ Furthermore, as advances are made, some conditions that were previously not felt to be surgically correctable can be totally or partially corrected with newer surgical techniques (for example, complex pulmonary atresia with major aortopulmonary collateral arteries and conversion of atrial switch to arterial switch).

In this study, patients fell into two groups: those with simple diagnoses (SASDs, repaired simple ventricular septal defects) made when they were adults or adults who have had primarily palliative procedures for complex conditions as children. In the third era there was an increase in patients undergoing surgery from previous "corrective" surgery, especially those having undergone total correction of tetralogy of Fallot (moderate complexity).

During the time period of this study, the total number of patients being operated on each year only slightly increased. However, the use of catheter interventions for atrial septal defect closure and coarctation relief has also increased with consequent decrease in the number of these patients being referred for surgery. For example, in our unit there were 93 catheter closures of SASDs from 1998-2001 alone.

The decrease in simple cases such as atrial septal defect closures has been offset by an increase in cases of moderate
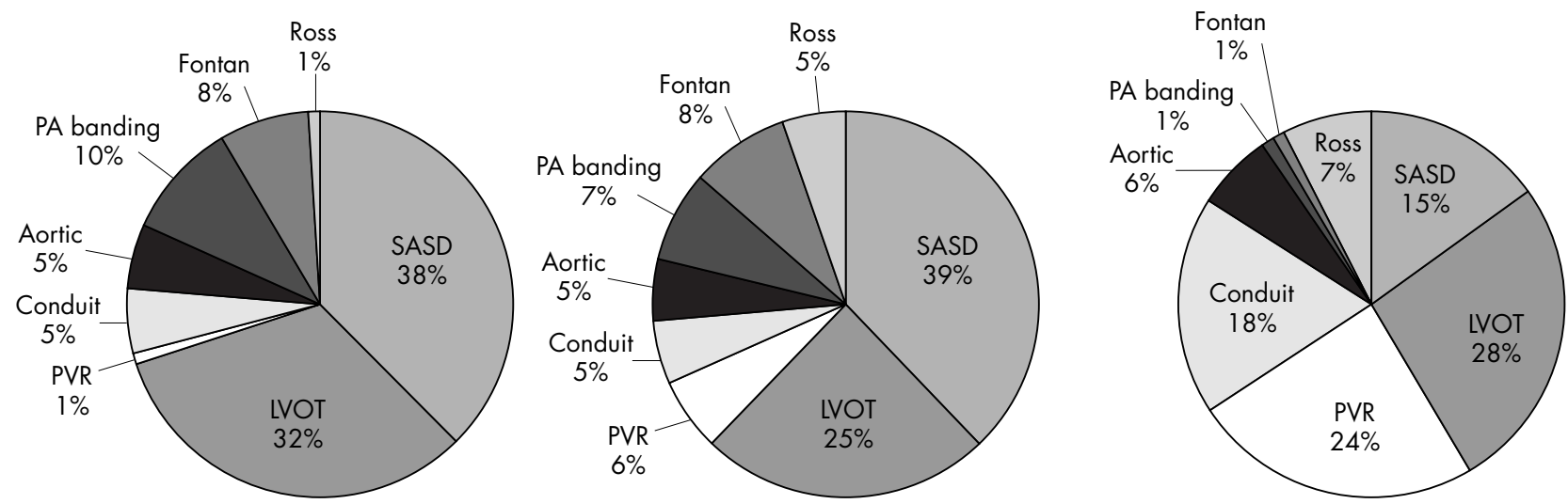

Figure 2 Distribution of procedures carried out over the three eras. Over the study eras there was a major increase in the proportion of patients undergoing the more complex procedures such as pulmonary valve replacement (PVR) and conduit replacements (for example, Rastelli). $\chi^{2} p<0.0001$ across eras. The differences are accounted for by the decrease in secundum atrial septal defects (SASD) and Fontan operations and the increases in PVR and conduit replacements in era 3 compared with both era 1 and era 2. There were less noticeable differences between era 1 and era 2. (appendix 2). Aortic, procedures isolated to the aorta itself, such as coarctation; Fontan, any type of Fontan-type operation; LVOT, left ventricular outflow tract procedures such as procedures for aortic stenosis; PA, pulmonary artery. 
Table 2 Comparison of procedure characteristics over three time periods

\begin{tabular}{|c|c|c|c|c|c|}
\hline & All eras & $\begin{array}{l}\text { Era } 1(1 / 1 / 90- \\
30 / 4 / 94)\end{array}$ & $\begin{array}{l}\text { Era } 2(1 / 5 / 94- \\
31 / 8 / 98)\end{array}$ & $\begin{array}{l}\text { Era } 3(1 / 8 / 98- \\
31 / 12 / 02)\end{array}$ & $p$ Value $^{*}$ \\
\hline $\begin{array}{l}\text { Bypass time (min) } \\
\text { Aortic cross clamp }\end{array}$ & $69.0(59.0)$ & $50.0(39.3))$ & $65.5(64.5)$ & $87.0(57.0)$ & $<0.001 \dagger \ddagger$ \\
\hline time (min) & $45.0(41.5)$ & $30.0(28.5)$ & $42.0(46.8)$ & $53.0(39.5))$ & $<0.001 \dagger \S$ \\
\hline ITU stay (nights) & $1(0.0)$ & $1(0.0)$ & $1(0.0)$ & $1(0.25)$ & $0.3906 \dagger$ \\
\hline Ventilation time (hours) & $8(10.3)$ & $7(10.0)$ & $7(10.8)$ & $9(10.6)$ & $0.0958 \dagger$ \\
\hline Blood use (units) & $1(2.0)$ & $1(3.0)$ & $1(2.0)$ & $1(2.0)$ & $0.7335 \dagger$ \\
\hline Deaths & $30(6.3 \%)$ & $12(7.3 \%)$ & $13(8.2 \%)$ & $5(3.4 \%)$ & 0.1847 \\
\hline All complications & $113(33 \%)$ & $26(23 \%)$ & $42(36 \%)$ & $45(41 \%)$ & 0.0931 \\
\hline Hospital stay (days) & $6(3.0)$ & $6(2.0)$ & $6(3.8)$ & $7(4.0)$ & $0.9370 \dagger$ \\
\hline \multicolumn{6}{|c|}{$\begin{array}{l}\text { Data are median (interquartile range) or number }(\%) \text {. } \\
\text { *For the differences across eras; } \dagger \chi^{2} \text { test; } \neq \text { analysis of variance; Sera } 1 v \text { era } 2, p=0.0014 \text {, era } 2 \text { vera } 3 \text {, } \\
p=0.0065 \text {, and era } 1 \text { vera } 3, p<0.001 \text {; } \uparrow \text { era } 1 \text { vera } 2, p=0.312 \text {, era } 2 \text { vera } 3, p=0.165 \text {, and era } 1 \text { era } 3 \text {, } \\
p<0.0001 \text {. } \\
\text { ITU, intensive treatment unit. }\end{array}$} \\
\hline
\end{tabular}

complexity such as tetralogy of Fallot, which account for a substantial increase in the number of pulmonary valve replacement procedures performed during this time. We can expect that this particular group will continue to increase in number. At present we have 140 patients in the GUCH programme who have moderate to severe pulmonary regurgitation and who we expect will require a pulmonary valve replacement in the future. A similar increase can be expected in the number of patients requiring conduit replacements.

The younger age of the patients with each era also reflects these trends. Increasing evidence for earlier pulmonary valve replacement in tetralogy of Fallot and the increasing number of children coming through into the adult clinics with conduits suggest that this trend in younger age at repeat operation may continue. ${ }^{5}{ }^{6}$ However, at the other end of the spectrum we are beginning to see acquired disease affecting the surgical procedures. Four patients in this series required a single coronary artery graft during surgery for their primary lesion (mean age 57 years, range 44-61 years). As the cohort of GUCH are followed up, it is inevitable that more acquired coronary and valvar disease will be seen, raising the question of whether these patients are best treated by the surgeons specialising in congenital or in acquired disease. A joint approach tailored to individual patient needs will undoubtedly be the solution.

Certainly, advances in catheter interventions may obviate the need for surgery in a proportion of these patients as more experience is gained with the use of catheter placed pulmonary valves. ${ }^{7}$ However, the maximum size of catheter delivered valves remains limiting and the medium term performance is still to be determined. It seems probable that many patients will still require surgery.

The operations have increased in complexity both by the nature of the patient populations (increased repeat procedures) and by increased use of technically demanding operations such as the Ross procedure. This is reflected in the increase in cardiopulmonary bypass times and aortic cross clamp times. However, despite the increased complexity of the cases, the mortality rate has decreased and hospital stay and ITU stay have remained constant. This partially reflects the youth of these patients and paucity of major co-morbidities such as concurrent lung disease, renal disease, diabetes, or atherosclerotic disease. Another important implication of the complexity of surgery is the increased demand for intraoperative echocardiography, which places additional demands on the cardiological support for these patients.

Although patients with lesions of moderate complexity have increased and patients with simple lesions have decreased, the proportion of patients with complex lesions who have undergone either complete repairs or palliative procedures has remained constant.
Although the number of complex Fontan-type procedures performed in adults has actually decreased (since the majority of Fontan completions are now performed in childhood), the failing Fontan is potentially a far greater medical problem. A small number of patients may have surgically treatable sequelae (for example, pathway stenosis) but there is also a considerable morbidity associated with the adult Fontan patients that requires specialist heart failure, electrophysiological, and transplant expertise. With close to 300 children in our region expected to graduate into the adult clinics over the next 10 years this has considerable implications for the GUCH medical and surgical service.

This aim of this study was to examine only patients with congenital heart disease who have undergone surgery. Most of the current literature deals either with specific lesions and their management or with the broad issues of managing adults with congenital heart disease. However, the limits of this study make it impossible to comment on the resource implications of the non-surgical requirements of these patients. This includes the interventional cardiology procedures, patients who have been referred for transplantation, and patients not yet requiring operation.

The data presented in this paper support the predictions of the British Cardiac Society working group that the surgical workload is likely to increase over the coming years-they have estimated a $22-30 \%$ increase by $2010 .^{8}$

The cost implications of providing services for these patients are important. The hospital cost of surgery for these patients is comparable with the costs for patients with acquired disease. The major cost of surgery is usually related to hospital stay, especially care in the ITU with the associated costs for blood transfusion, antibiotics and other drugs, equipment, and personnel costs. In this study, the patient's length of stay was comparable with that for patients undergoing CABG in the UK, the majority of whom spend between 6-10 days in hospital. ${ }^{9}$ Although the use of valves and conduits has an impact on total costs (about $£ 1500$ for one valve), it is important for future planning of GUCH services that we recognise that the costs of surgery can be comparable with costs of CABG surgery.

In this centre the adult congenital heart disease programme has become centralised and more formalised with the appointment of a GUCH consultant in cardiology in 2000. The development of a dedicated adult congenital service with streamlined transition of care from childhood into adolescence and adulthood has become the ideal type of care and has been strongly advocated by doctors and patient groups alike. ${ }^{8}{ }^{10}$ These specialised units need a multidisciplinary team approach that is centred on the adult congenital heart disease cardiologists and surgeons with direct access to electrophysiology, 
transplantation, radiology, and interventional cardiology expertise. Patients need dedicated facilities that can accommodate their requirements in "young adult units" with trained nursing and liaison nursing staff. ITUs and cardiac operating departments need to be trained and educated by the GUCH specialists to recognise the special requirements of these patients.

\section{Conclusions}

It is clear that adults with congenital heart disease are increasing in numbers and in complexity. Surgery for adult congenital heart disease is not decreasing despite the impact of interventional cardiology. The percentage of repeat surgery and complex diagnoses is increasing with correspondingly longer cardiopulmonary bypass times. Nevertheless, these patients continue to do well with a low mortality and hospital stay and cost implication that compare favourably with those for CABG surgery.

\section{Authors' affiliations}

S K Srinathan, B Sethia, W J Brawn, D J Barron, Birmingham Children's Hospital, Birmingham, UK

R S Bonser, S A Thorne, The Queen Elizabeth Hospital, Birmingham, UK

\section{APPENDIX 1}

\section{CLASSIFICATION OF DISEASE COMPLEXITY:}

\section{TABLES 3-5}

Data from Warnes et al. ${ }^{3}$

Table 3 Types of adult patients with simple congenital heart disease: patients who can usually be cared for in the general medical community

\section{Native disease}

Isolated congenital aortic valve disease

Isolated congenital mitral valve disease (except parachute valve, cleft

leaflet)

Isolated patent foramen ovale or small atrial septal defect

Isolated small ventriculoseptal defect (no associated lesions)

Mild pulmonary stenosis

Repaired conditions

Previously ligated or occluded patent duct arteriosus

Repaired secundum or sinus venosus atrial septal defect without residua Repaired ventriculoseptal defect with residua

Table 4 Types of adult patients with congenital heart disease of moderate severity: patients who should be seen periodically at adult congenital heart centres

\begin{tabular}{l}
\hline Aorto-left ventricular fistulae \\
Anomalous pulmonary venous drainage, partial or total \\
Atrioventricular canal defects (partial or complete) \\
Coarctation of the aorta \\
Ebsteins's anomaly \\
Infundibular right ventricular outflow obstruction of significance \\
Ostium primum atrial septal defect \\
Patent ductus arteriosus (not closed) \\
Pulmonary valve regurgitation (moderate to severe) \\
Sinus of Valsalva fistula/aneurysm \\
Sinus venosus atrial septal defect \\
Subvalvar or supravalvar aortic stenosis (except HOCM) \\
Tetralogy of Fallot \\
Ventricular septal defect with absent valve or valves \\
Aortic regurgitation \\
Coarctation of the aorta \\
Mitral disease \\
Right ventricular outflow tract obstruction \\
Straddling tricuspid/mitral valve \\
Subaortic stenosis \\
\hline HOCM, hypertrophic obstructive cardiomyopathy.
\end{tabular}

Table 5 Types of adult patients with congenital heart disease of great complexity: patients who should be seen regularly at adult congenital heart centres

Conduits, valved or non-valved

Cyanotic congenital heart (all forms)

Double outlet ventricle

Eisenmenger's syndrome

Fontan procedure

Mitral atresia

Single ventricle (double inlet or outlet, common or primitive)

Pulmonary atresia (all forms)

Pulmonary vascular obstructive disease

Transposition of the great arteries

Tricuspid atresia

Truncus arteriosus/hemitruncus

Other abnormalities of atrioventricular or ventriculoarterial connection not listed above (crisscross heart, isomerism, heterotaxy syndromes,

ventricular inversion)

\section{APPENDIX 2}

\section{CONTINGENCY TABLES AND $\chi^{2}$ VALUES: TABLES 6-8}

Table 6 Change in NYHA class across eras (table 1): $\chi^{2}$ cells

\begin{tabular}{llll}
\hline & Era 1 & Era 2 & Era 3 \\
\hline NYHA class & & & \\
I & 3.619 & 0.713 & 0.720 \\
II & 3.039 & 0.097 & 1.531 \\
III & 1.462 & 0.074 & 0.644 \\
IV & 0.620 & 0.023 & 0.300 \\
\hline
\end{tabular}

$\chi^{2}=12.842$ with $\mathrm{df}=6$

$\chi^{2} p=0.0456$.

NYHA, New York Heart Association.

Table 7 Change in the complexity of lesions across eras (fig 1): $\chi^{2}$ cells

\begin{tabular}{llll}
\hline & Era 1 & Era 2 & Era 3 \\
\hline Simple & 2.345 & 0.211 & 4.312 \\
Moderate & 1.423 & 0.367 & 3.511 \\
Complex & 0.246 & 0.011 & 0.169 \\
\hline \multicolumn{3}{l}{$\chi^{2}=12.595$ with $\mathrm{df}=4}$. \\
$\chi^{2} \mathrm{p}=0.0134$.
\end{tabular}

Table 8 Change in specific procedures across eras (fig 2): $\chi^{2}$ cells

\begin{tabular}{llll}
\hline & Era 1 & Era 2 & Era 3 \\
\hline SASD & 1.732 & 1.894 & 7.251 \\
LVOT & 0.711 & 0.409 & 0.038 \\
PVR & 7.995 & 1.668 & 16.900 \\
Conduit & 1.712 & 1.844 & 7.111 \\
Aortic & 0.014 & 0.028 & 0.083 \\
PA banding & 2.054 & 0.283 & 3.843 \\
Fontan & 0.563 & 1.264 & 3.521 \\
Ross & 2.520 & 0.088 & 1.641 \\
\hline
\end{tabular}

$\chi^{2}=65.169$ with $\mathrm{df}=14$

$\chi^{2} \mathrm{p}<0.0001$.

LVOT, left ventricular outflow tract; PA, pulmonary artery; PVR, pulmonary valve replacement; SASD, secundum atrial septal defect. 


\section{REFERENCES}

1 British Cardiac Society. Report of the British Cardiac Society Working Party. Grown-up congenital heart (GUCH) disease: current needs and provision of service for adolescents and adults with congenital heart disease in the UK. Heart 2002;88(suppl I):i1-14.

2 Webb GD, Williams RG. Care of the adult with congenital heart disease: introduction. J Am Coll Cardiol 2001;37:1166.

3 Warnes CA, Liberthson R, Danielson GK, et al. Task Force 1: the changing profile of congenital heart disease in adult life. J Am Coll Cardiol 2001;37:1170-5.

4 Perloff JK, Warnes CA. Challenges posed by adults with repaired congenital heart disease. Circulation 2001;103:2637-43.

5 Therrien J, Siu SC, McLaughlin PR, et al. Pulmonary valve replacement in adults late after repair of tetralogy of Fallot: are we operating too late? J Am Coll Cardiol 2000;36:1670-5.
6 Therrien J, Siu SC, Harris L, et al. Impact of pulmonary valve replacement on arrhythmia propensity late after repair of tetralogy of Fallot. Circulation 2001;103:2489-94.

7 Khambadkone S, Boudjemline Y, Franklin O, et al. Percutaneous pulmonary valve implantation for right ventricular outflow tract lesions after congenital heart surgery [abstract]. Cardiol Young 2003;13(suppl 1):32.

8 Anon. Report of the Paediatric and Congenital Cardiac Services Review Group. December 2003. www.advisorybodies.doh.gov.uk/childcardiac/ pccsreptdec03.pdf (accessed 3 April 2004).

9 Keogh BE, Kinsman R. National adult cardiac surgical database report 20002001. London: The Society of Cardiothoracic Surgeons of Great Britain and Ireland, 2002.

10 Therrien J, Dore A, Gersony W, et al. CCS consensus conference 2001 update: recommendations for the management of adults with congenital heart disease. Part 1. Can J Cardiol 2001;17:940-59.

\section{IMAGES IN CARDIOLOGY}

\section{Pulmonary artery obstruction mimicking the clinical features of acute pulmonary embolism}

A 44 year old woman, who had enjoyed good health up until recently, presented with a three month history of progressive shortness of breath and bilateral ankle oedema. Physical examination revealed tachycardia of 125 beats per minute and a blood pressure of 100/60 $\mathrm{mm} \mathrm{Hg}$ on admission. Elevated jugular venous pressure with giant $\mathrm{CV}$ wave pattern was observed at the level of the earlobe. Hepatomegaly and bilateral pitting ankle oedema were also noted on palpation. There was a soft, grade $2 / 6$ ejection systolic murmur best heard over the pulmonary area. The oxygen saturation was about $95 \%$ on room air. Chest $x$ ray showed cardiomegaly and widened mediastinum. ECG revealed sinus tachycardia, right axis deviation and $S_{I} Q_{I I I}$ $\mathrm{T}_{\text {III }}$ pattern (below left). The clinical presentation and the ECG pattern were typical of acute pulmonary embolism. Computed tomography with contrast of the thorax strikingly showed no evidence of pulmonary embolism but a huge, solid mediastinal tumour encroaching on the heart. The main pulmonary artery and the right ventricular outflow tract was grossly compressed and squeezed, leaving only a slit-like lumen (below right). The entire heart was pushed aside towards the left hemithorax. Serum $\alpha$-fetal protein $(\alpha \mathrm{FP})$ and human chorionic gonadotrophin (HCG) concentration were

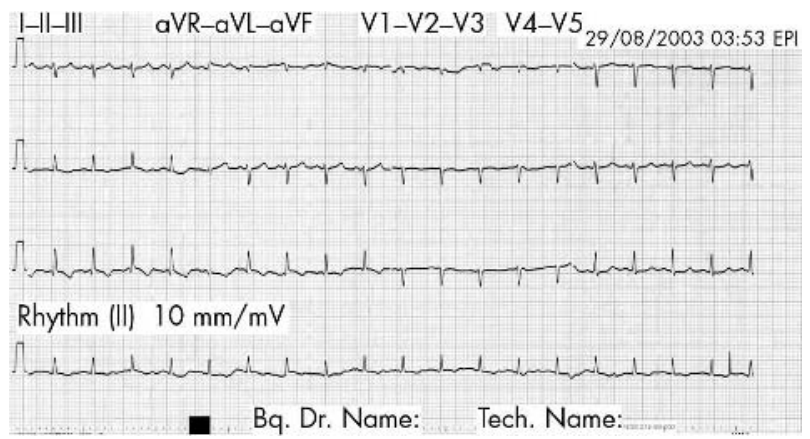

normal. Mediastinoscopy and biopsy of the tumour was subsequently performed which yielded malignant spindle cell tumour by histology. In view of the probable invasion of the heart and great vessels by the tumour, complete curative surgical resection would not be possible. Chemo-irradiation was offered to the patient as an alternative but she finally declined this treatment option after knowing and understanding all the side effects of the treatment and the prognosis of her disease.

Spindle cell tumour is a rare disease of mesenchymal origin. This case is unusual because this rapid growing tumour caused external compression and luminal obstruction of the main pulmonary trunk, the latter being exactly the same pathological mechanism as pulmonary embolism.

C W Siu
M-H Jim
H F Tse
hftse@hku.hk

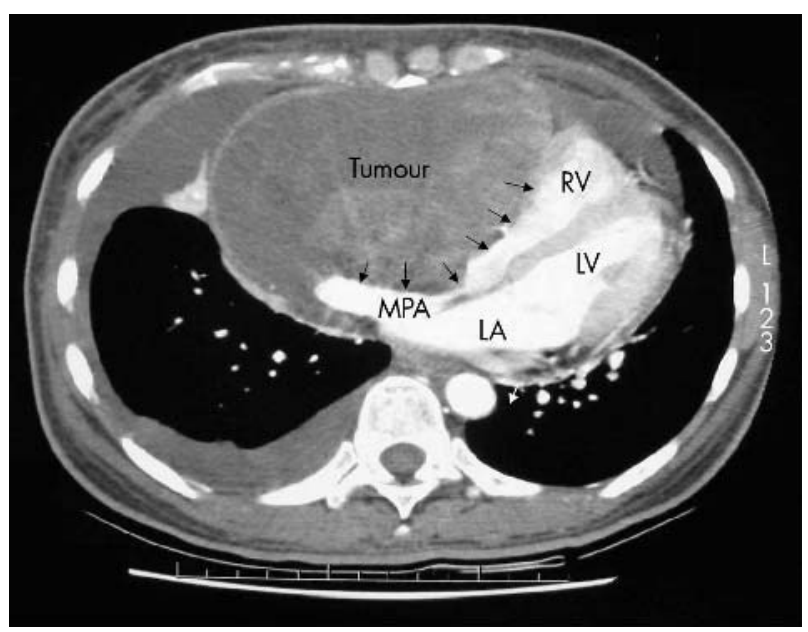

